\title{
Perinatal Transmission of Dengue: A Report of 7 Cases
}

\author{
Christiane Fernandes Ribeiro, $\mathrm{PhD}^{1}$, Vânia Glória Silami Lopes, $\mathrm{PhD}^{2}$, Patrícia Brasil, $\mathrm{PhD}^{3}$, Janice Coelho, $\mathrm{MD}^{3}$, \\ Adriana Gouveia Muniz ${ }^{3}$, and Rita Maria Ribeiro Nogueira, $\mathrm{PhD}^{4}$
}

Perinatal transmission of dengue virus was confirmed by the evidence of virus in fetal tissue, newborn serum, and placenta of pregnant women. Abortion, several different clinical findings, and placental inflammatory findings were documented. No association was seen between severity of maternal dengue and disease of the newborn. (J Pediatr 2013;163:1514-6).

engue, currently the most widespread human viral disease transmitted by mosquitoes, is endemic in many tropical countries. The dengue virus (DENV) is classified into 4 serotypes (1, 2, 3, and 4). Since 1948, the occurrence of dengue in pregnancy has been reported in the literature, mostly in case reports. The largest series comprised 38 cases in 2000 and 53 in 2009, both in French Guiana. ${ }^{1}$ Maternal deaths attributed to dengue during pregnancy recently have been described in Brazil. ${ }^{2}$ Perinatal transmission of DENV in pregnancy was confirmed either by DENV isolation or by the presence of specific IgM antibodies in newborns. ${ }^{3-5}$ We report 7 confirmed cases of DENV infection acquired through perinatal transmission.

\section{Methods}

From January 2008 to July 2010, 28 placentas and 7 ovular remnants from pregnant women with laboratoryconfirmed dengue were analyzed by the pathology service of the University Hospital Antônio Pedro, Niterói, Rio de Janeiro, Brazil. This study was approved by the Ethics Committee of the Hospital Universitário Antônio Pedro, Universidade Federal Fluminense (CMM/HUAP No. 038).

Laboratory confirmation of dengue was defined as the presence of IgM by the method of IgM antibody capture enzyme-linked immunosorbent assay, by the presence of nucleic acid by reverse-transcription polymerase chain reaction in maternal or neonatal serum, or by immunohistochemistry in placental tissue.

Placentas and ovular remnants were analyzed by macroscopic examination after fixation in $10 \%$ formalin. Cleavage was performed, and 7 samples were retrieved from the placenta and annexes. Samples were subsequently stained with hematoxylin-eosin. The ovular membranes, umbilical cord, and placental tissue were analyzed histologically. Immunohistochemistry was conducted with the murine IgG2A monoclonal anti-DENV complex (MAB8705; clone D3-2H2-9-21; Millipore, Billerica, Massachusetts) diluted 1:300 in phosphate-buffered saline and incubated overnight.

$\begin{array}{ll}\text { DENV } & \text { Dengue virus } \\ \text { DHF } & \text { Dengue hemorrhagic fever } \\ \text { DSS } & \text { Dengue shock syndrome }\end{array}$

Paraffinized liver tissue from a patient who died from DENV was used as the positive control for the immunohistochemical analysis. For the negative immunohistochemical control, 2 different procedures were incorporated: (1) paraffinized liver tissue from the patient who had died of dengue, suppressing the monoclonal antibody, the primary antibody of the incubation step, using only the diluent solution (bovine serum albumin) and (2) paraffin-embedded placental tissue of patients without infectious disease on which the full technique was performed (without the deletion of the MAB). ${ }^{6,7}$

\section{Results}

Maternal, fetal, and neonatal clinical findings and outcomes are described. Perinatal transmission was confirmed in 7 cases. In 5 cases, the DENV infection was confirmed by immunohistochemistry by the presence of marking in the decidua or placenta villus. In 4 of these, villous stromal edema, excessive formation of syncytial knots, and chorangiosis were the main pathologic findings of placental hypoxia. DENV also was demonstrated in 1 case by immunohistochemistry in the epithelial cells of lung, ependymal, and kidney of the aborted fetus (Figure).

The time elapsed between the first day of maternal disease (D1) and date of birth or abortion ranged from 1 to 8 days, with an average of 3.4 days and median of 3 days. No newborn presented with asphyxia during delivery. Only 1 newborn presented with low birth weight. Maternal, fetal, and newborn clinical manifestations, laboratory findings, dengue diagnosis, and outcomes are illustrated in the Table.

\section{Discussion}

This report of perinatal transmission of dengue documents the presence of DENV in fetal tissue in which the pregnant

From the ${ }^{1}$ Departments of Materno-infantil and ${ }^{2}$ Pathology, Universidade Federal Fluminense; ${ }^{3}$ Institute of Clinical Research Evandro Chagas/Fiocruz; and ${ }^{4}$ Flavirus Laboratory, Oswaldo Cruz Institute/Fiocruz, Rio de Janeiro, Brazil

Funded by Fundo de Amparo à Pesquisa do Estado do Rio de Janeiro (Fund for Research Support of the State of Rio de Janeiro; 304872/2011-13) and Conselho Nacional de Pesquisa (National Research Council; E-26/103.149/2011). The authors declare no conflicts of interest.

0022-3476/\$ - see front matter. Copyright $(2013$ Mosby Inc.

All rights reserved. http://dx.doi.org/10.1016/j.jpeds.2013.06.040 


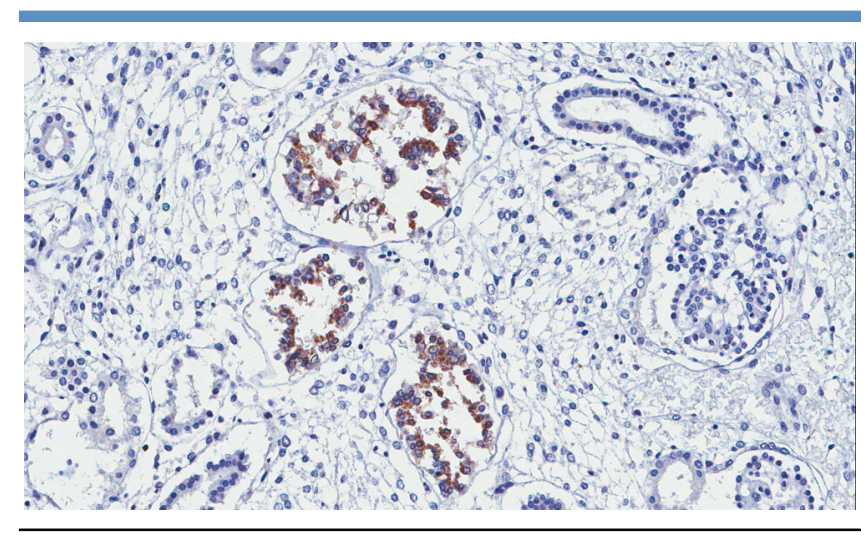

Figure. Microscopy kidney of aborted fetus. Glomerular and tubular structures expressing positive immunoreaction to DENV (20x).

women were diagnosed with dengue. DENV also was confirmed in the placenta and newborn serum.

A spectrum of dengue clinical presentations was observed in newborns, from asymptomatic (cases 2 and 6) to dengue hemorrhagic fever (DHF) or dengue shock syndrome (DSS) (cases 3 and 4, respectively), as has been previously described. ${ }^{4,8}$ In 3 cases, the newborns were mildly symptomatic (cases 2,6 , and 7), showing only a nonspecific laboratory change such as leukopenia, thrombocytopenia, or hemoconcentration that did not correspond to the mother's severity of dengue. No instances of premature birth occurred, possibly because of the onset of maternal infection between 37 and 39 weeks of pregnancy. However, abortion occurred in 1 case of infection during the first trimester of pregnancy (12th week), confirming recent findings. ${ }^{9}$

Infants who developed DHF or DSS (cases 3 and 4) and the single case of abortion (case 5) had a severe inflammatory response and premature detachment of the placenta, reflecting a more severe course of the disease. However, there was no relationship between severity of newborn/fetal outcome and the mother's clinical presentation of dengue. DENV was identified in all but 2 cases of neonatal infection, with IgM present in the serum after 1 day of maternal disease. These findings indicate that neonatal DENV infection occurred during the incubation period of the mother's disease.

A period of 1 to 8 days between the first day of mother's illness and childbirth was sufficient to cause DENV perinatal transmission and harm to the fetus. This finding suggests that the virus itself might be the major factor responsible for the injury of the concept in pregnant DENV-infected women. However, it is not known whether subneutralizing levels of maternally derived anti-DENV IgG serotypes enhanced DENV-2 infection in neonatal Fc receptor-bearing cells and therefore contributed to severity of the newborn diseases.

The most severe clinical newborn cases ( 3 and 4 ) responded favorably to intensive care and antibiotic therapy. In the cases of neonatal DHF/DSS (cases 3 and 4), an association of severity with neonatal bacterial infection cannot be excluded. The differentiation of dengue in the neonatal period from neonatal bacterial sepsis is difficult, and in this context, specific

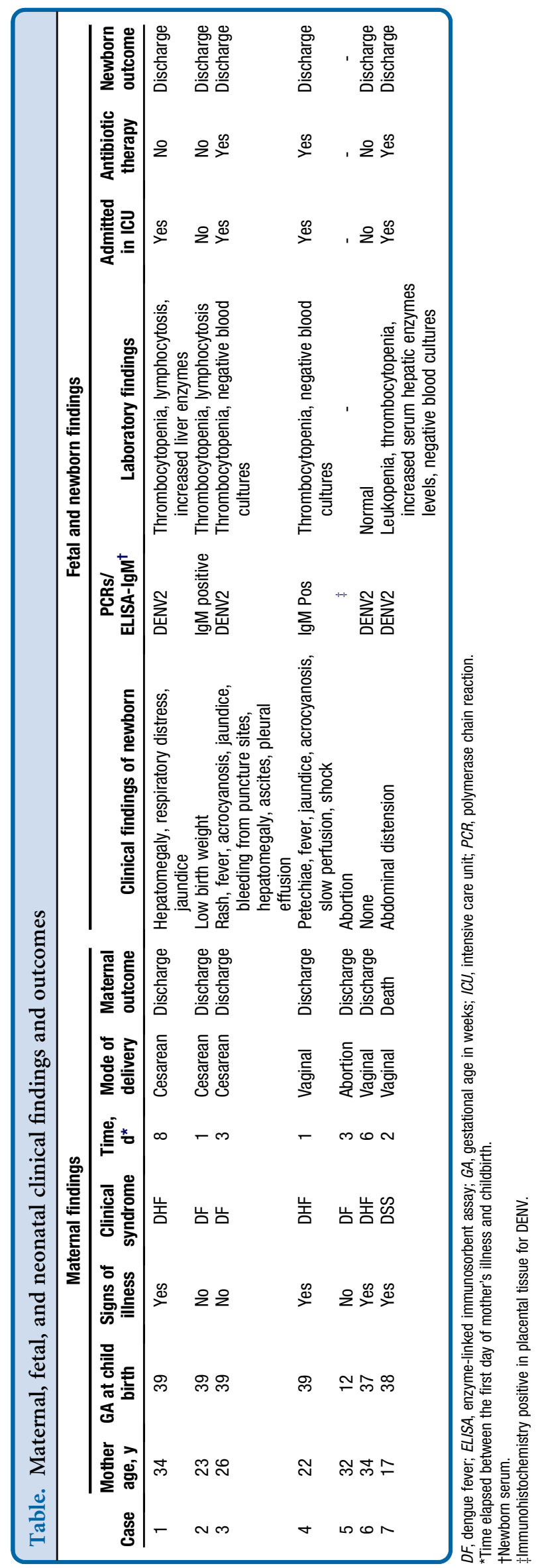


treatment for bacterial sepsis may have contributed to a better prognosis of severe neonatal disease. ${ }^{4,10}$ Dengue should be included in the differential diagnosis of neonatal sepsis in hyperendemic areas such as Brazil, as well as for children of pregnant women who visit areas where dengue is endemic.

We acknowledge Pedro Henrique Fernandes Josephson Ribeiro, and Gustavo Nascimento for their contribution in data collection. EDITAGE reviewed and revised the English for this manuscript.

Submitted for publication Jan 17, 2013; last revision received Apr 19, 2013; accepted Jun 19, 2013.

Reprint requests: Christiane Fernandes Ribeiro, PhD, Departamento Maternoinfantil, Universidade Federal Fluminense,303, Marquês do Paraná Street, $3^{\circ}$ floor, Centro, Niterói, Rio de Janeiro 24033-900, Brazil. E-mail: chrisribeiro@ vm.uff.br

\section{References}

1. Carles G, Talarmin A, Peneau C, Bertsch M. Dengue fever and pregnancy. A study of 38 cases in French Guiana. J Gynecol Obstet Biol Reprod (Paris) 2000;29:758-62.
2. Alvarenga C, Silami V, Brasil P, Boechat ME, Coelho J, Nogueira RM. Dengue during pregnancy: a study of thirteen cases. Am J Infect Dis 2009;5:295-300.

3. Boussemart T, Babe P, Sibille G, Neyret C, Berchel C. Prenatal transmission of dengue: two new cases. J Perinatol 2001;21:255-7.

4. Chye JK, Lim CT, Ng KB, Lim JM, George R, Lam SK. Vertical transmission of dengue. Clin Infect Dis 1997;25:1374-7.

5. Janjindamai W, Pruekprasert P. Perinatal dengue infection: a case report and review of literature. Southeast Asian J Trop Med Public Health 2003; 34:793-6.

6. Dabbs DJ. Diagnostic immunohistochemistry. Edinburgh: Churchill Livingstone Elsevier; 2006.

7. Salgado DM, Eltit JM, Mansfield K, Panqueba C, Castro D, Vega MR, et al. Heart and skeletal muscle are targets of dengue virus infection. Pediatr Infect Dis J 2010;29:238-42.

8. Basurko C, Carles G, Youssef M, Guindi WEL. Maternal and fetal consequences of dengue fever during pregnancy. Eur J Obstet Gynecol Reprod Biol 2009;147:29-32.

9. Chau TNB, Hieu NT, Anders KL, Wolbers M, Lien LB, Hieu LTM, et al. Dengue virus infections and maternal antibody decay in a prospective birth cohort study of Vietnamese infants. J Infect Dis 2009;200:1893-900.

10. Edmond K, Zaidi A. New approaches to preventing, diagnosing, and treating neonatal sepsis. PLoS Med 2010;7:e1000213. 